Lorenzo Dal Ri / Umberto Tecchiati

\title{
NUOVI INDIZI DI POPOLAMENTO PREISTORICO IN VAL BADIA
}

Presentiamo in questo breve contributo alcuni rinvenimenti effettuati a più riprese ad opera del geologo H. Buratti in località Piz Störes presso Prelungé (Alta Val Badia) a $2170 \mathrm{~m}$ di quota. Trattasi di una cuspide di freccia frammentaria (fig. 2.1) (manca la punta, il codolo e parte delle alette) in selce nocciola con piccole

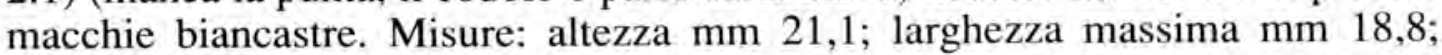
spessore $\mathrm{mm} \mathrm{4,5}$. La parte superiore del manufatto presenta margini leggermente convessi che si allargano in prossimità delle alette conferendo al profilo un aspetto sinuoso. Il ritocco foliato coprente, bifacciale, presenta su una delle facce stacchi piatti che convergono paralleli dai margini verso l'asse longitudinale del manufatto, disponendosi in modo subperpendicolare ad esso. L'altra faccia presenta un ritocco meno regolare. Il profilo dei margini taglienti è ben rettilineo. La forma del manufatto e la tipologia del ritocco, che lo avvicina alla tecnologia delle lame di pugnale in selce, consentono una datazione ad un momento iniziale o evoluto dell'età del rame (prima metà del III millennio a.C.).

Un secondo manufatto (fig. 2,2), un grattatoio frontale a ritocco erto, su selce biancastra, sembra doversi riferire a momenti più arcaici di frequentazione del sito (mesolitico antico, intorno all'VIII-VII millennio a.C.). Il manufatto, frammentario, misura mm 17 di lunghezza massima, mm 19 di larghezza massima e mm 3 di spessore.

Si deve ancora ad una raccolta di superficie, questa volta ad opera del Prof. R. Rottonara di La Villa, la scoperta di un acciarino su spessa lama in selce biondogrigiastra. Il manufatto, che non escludiamo essere stato realizzato a partire da un manufatto preistorico, forse neolitico, misura mm 44,9 di lunghezza, $\mathrm{mm} \mathrm{17,5} \mathrm{di}$ larghezza e $\mathrm{mm} 7$ di spessore.

Il reperto alla fig. 2.1 rientra nel novero degli oggetti sporadici dell'età del rame raccolti in quota in numerose località della regione ${ }^{1)}$ e prova che il territorio dolomitico e in particolare il versante più solatio della Val Badia, fino a questo momento assai povero di reperti ${ }^{2}$, era frequentato, oltre che nel mesolitico, come prova il grattatoio alla fig. 2.2. anche in epoca postmesolitica da cacciatori e/o pastori della prima età dei metalli. La prossimità geografica del luogo di rinveni-

1) cfr. Bagolini \& Pedrotti, 1992,"Vorgeschichtliche Höhenfunde im TrentinoSüdtirol und im Dolomitenraum vom Spätpaläolithikum bis zu den Anfängen der Metallurgie", in: AA.VV., Der Mann im Eis, Innsbruck, pp 359-377.

2) cfr. Bagolini \& Tecchiati, 1993a, "Os- servazioni sul popolamento delle valli ladine tra neolitico ed età del bronzo nel quadro della preistoria del bacino atesino", in: AA.VV., Archeologia nelle Dolomiti. Ricerche e ritrovamenti nelle valli del Sella dall'età della pietra alla romanità, pp 47-56. 


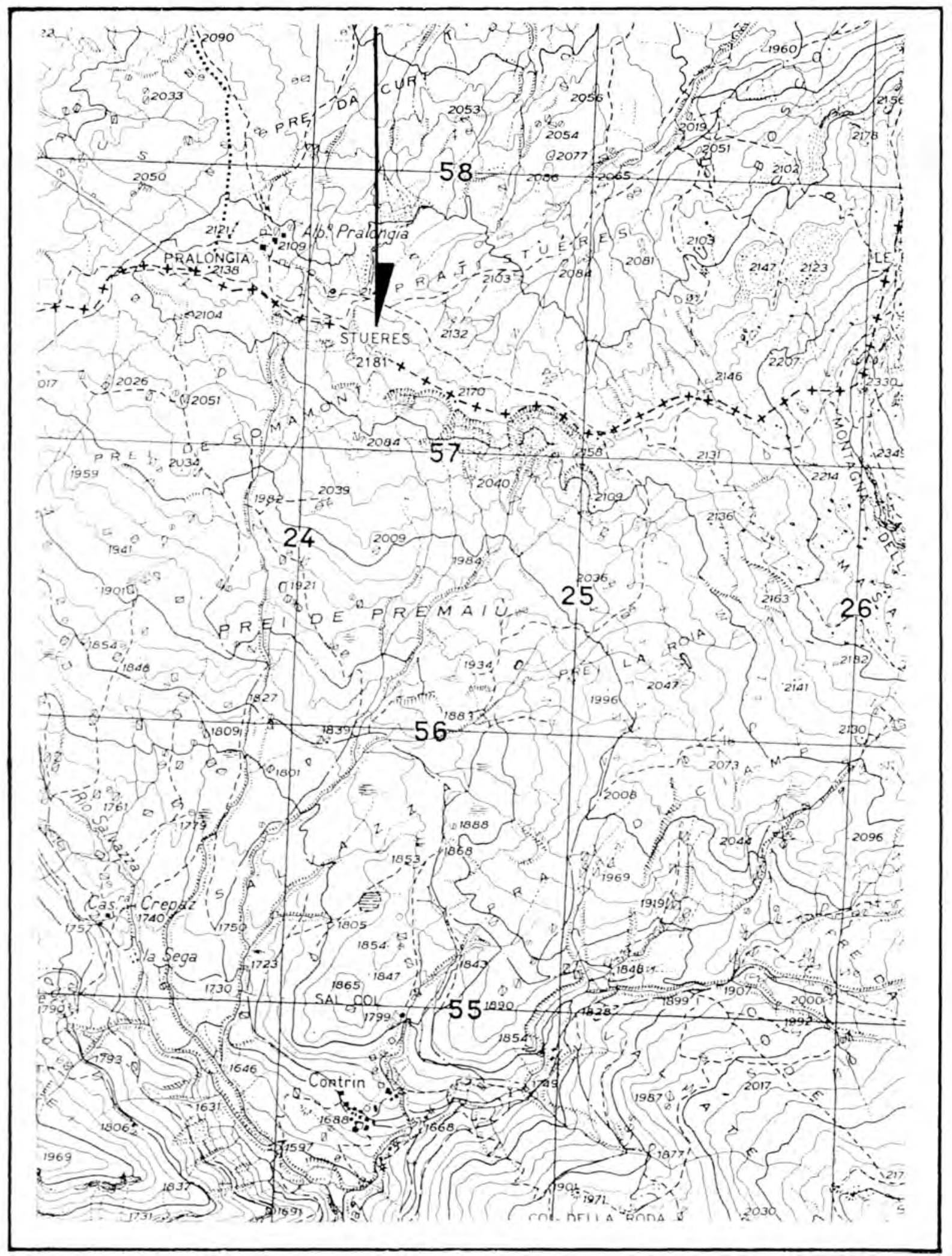

Fig. 1: Ubicazione del sito sulla tavoletta 1:25.000 "Corvara in Badia", F 11, I, S.E. 


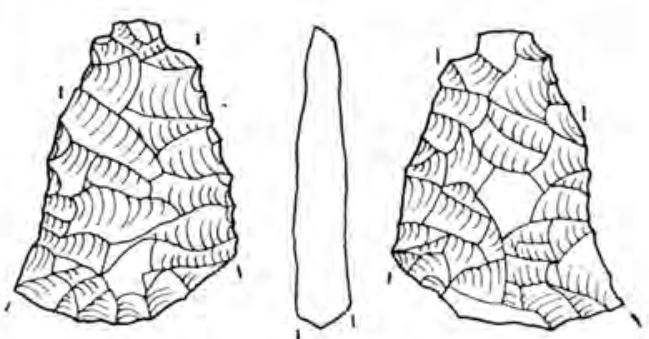

SAN CASSIANO

Prelungé

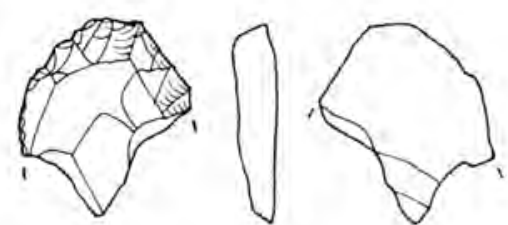

SAN CASSIANO

Prelungé
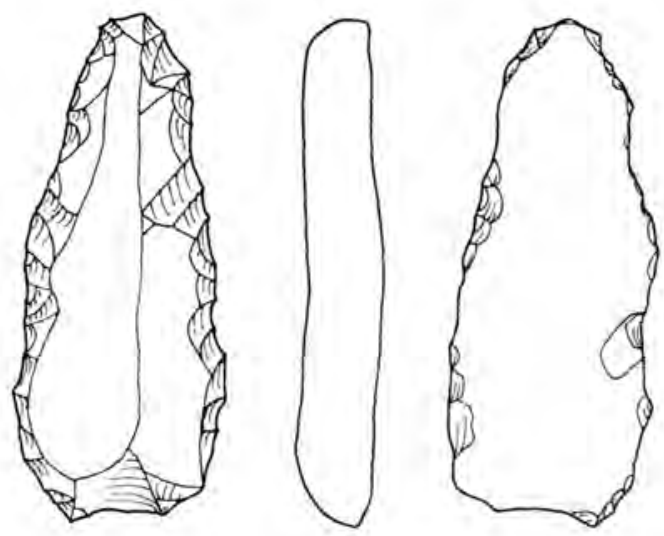

0 $2 \mathrm{~cm}$

SAN CASSIANO

Prelungé

Fig. 2: 1) Punta di freccia frammentaria eneolitica; 2) grattatoio frammentario mesolitico; 3) acciarino moderno (riuso di manufatto preistorico forse neolitico). Disegni di G. Fusi-Bolzano. 




Fig. 3: Il manufatto in pietra rinvenuto in località "Curt" presso Pieve di Marebbe. 
mento dei manufatti qui descritti, rispetto all'abitato su altura di Sotćiastel (San Leonardo $)^{3)}$, propone la suggestiva ipotesi che l'insediamento della media e tarda età del bronzo si sia sviluppato lungo una via di transito secondaria ma frequentata, che immetteva attraverso i passi alpini di Valparola e Falzarego e la valle del Cordevole al bacino del Piave e alla pianura Padana - in un territorio non nuovo a forme di presenza umana legate principalmente allo sfruttamento agro-pastorale.

Riveste ancora qualche interesse il ritrovamento di un pesante percussore litico avvenuto ad opera della Prof.ssa T. Palfrader-Willeit in località "Curt" presso Pieve di Marebbe. Trattasi di un oggetto che attirò l'attenzione della scopritrice per la forma insolita e per la levigatezza delle superfici. Mentre forma e politezza dell'oggetto sono del tutto naturali, le tracce di uso che esso presenta alle estremità, riconducibili ad un' attività di percussione, avvicinano il manufatto di Curt ai molti percussori noti in diversi ambiti preistorici e protostorici. Un inquadramento più preciso è impossibile a causa della mancanza di un contesto archeologico di riferimento e di associazioni cronologicamente e culturalmente note, in un sito che, nonostante le promettenti caratteristiche morfologiche e la favorevole posizione geografica non ha restituito finora resti di interesse archeologico ${ }^{4)}$.

3) cfr., con relativa bibliografia, Bagolini \& Tecchiati, 1993b, "Sotciastel: un abitato fortificato della media e recente età del bronzo in Val Badia (Comune di San Leonardo)", in: AA.VV., Archeologia nelle Dolomiti. Ricerche e ritro-

vamenti nelle valli del Sella dall'età della pietra alla romanità, pp 87-93

4) cfr. Tecchiati, U., 1992, "Nuove ricerche sul popolamento preistorico e protostoricodi Val Badiae Val Gardena, in: Ladinia $X V I$, pp. 101-127. 